\title{
The Cambridge MRI database for animal models of Huntington disease
}

Stephen J. Sawiak ${ }^{1,2,3^{*}}$ and A. Jennifer Morton ${ }^{3}$

${ }^{1}$ Wolfson Brain Imaging Centre, University of Cambridge, Box 65 Addenbrooke's Hospital, Hills Road, Cambridge, CB2 0QQ

${ }^{2}$ Behavioural and Clinical Neuroscience Institute, University of Cambridge, Downing Street, Cambridge

${ }^{3}$ Physiology, Development and Neuroscience, University of Cambridge, Downing Street, Cambridge

*Corresponding author

Dr Stephen J Sawiak

Wolfson Brain Imaging Centre

University of Cambridge

Box 65, Addenbrooke's Hospital

Hills Road

Cambridge

United Kingdom

CB2 OQQ

tel +44 1223746463

sjs80@cam.ac.uk

\section{Abstract}

We describe the Cambridge animal brain magnetic resonance imaging repository comprising 400 datasets to date from mouse models of Huntington disease. The data include raw images as well as segmented grey and white matter images with maps of cortical thickness.

All images and phenotypic data for each subject are freely-available without restriction from http://www.dspace.cam.ac.uk/handle/1810/243361/). Software and anatomical population templates optimised for animal brain analysis with MRI are also available from this site.

\section{Keywords}

R6/2, mouse models, sheep models 


\section{Introduction}

Huntington's disease (HD) is a fatal, progressive neurodegenerative disorder with no known cure and few effective treatments. It is caused by a single dominant genetic mutation, that is an expanded polyglutamine CAG repeat in the coding region of the $H T T$ gene. Expression of the mutant gene causes changes in cellular function which lead to symptoms that include chorea, progressive psychiatric disturbances and cognitive impairment. The disease affects 4-10 persons per 100,000 in Europe and North America. A specific genetic test allows pre-clinical diagnosis years before the onset of unequivocal signs of disease (Walker, 2007).

Progress towards effective therapies relies upon the availability and use of appropriate animal models that can be used for experiments to understand disease pathophysiology. The most widely used animal model of HD is the R6/2 mouse which carries a fragment of the gene responsible for HD. We have studied extensively the R6/2 mouse with high-resolution MRI and voxel- and tensor-based morphometry (Sawiak et al., 2009a, b, 2013).

In R6/2 mice, as is seen in human patients, the number of CAG repeats in the mutant gene correlates with disease onset and severity. It appears, however, that extremely large repeat lengths (>300 CAG repeats) lead to a delayed phenotype in the R6/2 mouse (Dragatsis et al., 2009; Morton et al., 2009). We have isolated colonies of R6/2 mice on a consistent background strain carrying CAG repeats between 110-460 repeats in size and imaged the brains of these using an identical high-resolution protocol. All of these images are available online, along with details of age, genotype and sex for researchers to download and use for any purpose (Sawiak et al., 2012). In addition to the R6/2 mice, we have included images from another mouse model commonly used for HD studies, the yeast artificial chromosome (YAC) mouse (Slow et al., 2003).

Some aspects of HD are difficult to model precisely in mice, due to differences in lifespan and anatomy (Morton and Howland, 2013). Recently an ovine model of HD has been assessed with MRI (Morton et al., 2014) and we are currently adding ovine datasets to our online data repository to supplement the mouse data.

Together, these animal brain images provide a powerful resource for studying the morphological phenotype of transgenic HD models and provide an open dataset for cross-species research.

\section{Repository details}

Our database is available at http://www.dspace.cam.ac.uk/handle/1810/243361.

The Cambridge HD MRI database was designed to enable the sharing of high-resolution animal model brain data for researchers to use for any purpose. The aim was that by making the data freely available online for those without access to these mouse strains or the resources to perform MRI, and also those with expertise that we lack to the benefit of the entire neuroimaging and HD communities. A secondary aim was that given there are very few large animal brain MRI repositories, our database could be useful as a common easily-accessed database of images for testing experimental methodology using animal brain data.

We are continuing to add to the database ad hoc. Thus it is not a static resource, but one which will incorporate all of the shareable animal brain data that we acquire. 
A breakdown of the mice available to date is shown in Figure 1. At present, there are data from 399 mice comprising reconstructed MRI images at $70 \mu \mathrm{m}$ isotropic resolution, segmented grey and white matter maps and cortical thickness maps for each animal. Along with each dataset, the genotype and background strain are available as a spreadsheet and listed on the website. Full details of the animals and the scan sequences used are described in (Sawiak et al., 2012) and (Morton et al., 2014). Typical images are shown in Figure 2.

The library also contains 6 OVT73 sheep (Jacobsen et al., 2010) with 6 matched control sheep. Five pairs of sheep are five years old and one pair is three years old. The sheep data were acquired in vivo with $0.6 \mathrm{~mm}$ isotropic resolution for T1-weighted images, $0.8 \mathrm{~mm}$ isotropic resolution for T2weighted and DTI data of $2 \mathrm{~mm}$ isotropic resolution (b-value $1000 \mathrm{~s} / \mathrm{mm}^{2}$ with 64 directions) A typical dataset from the library is shown in Figure 3.

All of our MRI data is shared in Nifti format (NIH http://nifti.nimh.nih.gov/nifti-1). The mouse data come from two different scanners (1T and 4.7T dedicated preclinical scanners) in our facility. Ex vivo sheep data were acquired in Cambridge using a Bruker BioSpec $4.7 \mathrm{~T} 40 \mathrm{~cm}$ bore scanner and in vivo sheep data were acquired at the Large Animal Research and Imaging Facility at 1.5T with a Siemens Sonata system (LARIF, Gilles Plains, Australia). None of the data has citable dois/uris.

Each MRI has been reviewed manually at acquisition and checked by us before it was uploaded to the database. Specifically, we checked for whole brain coverage, coil non-uniformity problems and signal to noise ratio or obvious imaging artefacts. Datasets with any of these issues were not included.

Access to the data is unrestricted through the University of Cambridge Data Repository (dSpace). No registration is required. All data ( $\mathrm{MRI}$ and phenotypic data) can be obtained via the same website.

We have no formal data usage agreement paperwork, but we ask the users to cite our work if they use our data. Since no registration is required, we have no system for alerting people to additions or changes in the repository.

The simplest way to download small numbers of datasets is via a simple web interface that exists for each file to be downloaded categorised by genotype. It is possible for experienced users to use a shell script to automate downloading of the data and we will help users do this via ftp if requested.

We have stated that we would be very pleased to host further data as part of our database to enrich. To date, however, we have not received any requests for inclusion of other datasets. Researchers interested in adding images to our database should email the corresponding author for instructions.

For data processing, we have written a toolbox for the SPM package (Wellcome Trust Centre for Neuroimaging, $\mathrm{UCL}$ ) with templates for animal brains and adjustments to the default settings to facilitate preclinical morphometric and functional data processing. The toolbox can be downloaded from link on the dSpace site or from http://www.spmmouse.org. 


\section{Acknowledgements}

This project is supported by CHDI Inc. We are grateful to Zhiguang Chang, Nigel Wood, Greg Brown and Skye Rudiger for assistance with acquiring the data in our library.

\section{References}

Dragatsis, I., Goldowitz, D., Del Mar, N., Deng, Y.P., Meade, C.A., Liu, L., Sun, Z., Dietrich, P., Yue, J., Reiner, A., 2009. CAG repeat lengths $>$ or $=335$ attenuate the phenotype in the R6/2 Huntington's disease transgenic mouse. Neurobiol Dis 33, 315-330.

Jacobsen, J.C., Bawden, C.S., Rudiger, S.R., McLaughlan, C.J., Reid, S.J., Waldvogel, H.J., MacDonald, M.E., Gusella, J.F., Walker, S.K., Kelly, J.M., Webb, G.C., Faull, R.L., Rees, M.I., Snell, R.G., 2010. An ovine transgenic Huntington's disease model. Hum Mol Genet 19, 1873-1882.

Morton, A.J., Glynn, D., Leavens, W., Zheng, Z., Faull, R.L., Skepper, J.N., Wight, J.M., 2009.

Paradoxical delay in the onset of disease caused by super-long CAG repeat expansions in R6/2 mice. Neurobiol Dis 33, 331-341.

Morton, A.J., Howland, D.S., 2013. Large genetic animal models of Huntington's Disease. J Huntingtons Dis 2, 3-19.

Morton, A.J., Rudiger, S.R., Wood, N.I., Sawiak, S.J., Brown, G.C., McLaughlan, C.J., Kuchel, T.R., Snell, R.G., Faull, R.L., Bawden, C.S., 2014. Early and progressive circadian abnormalities in Huntington's disease sheep are unmasked by social environment. Hum Mol Genet 23, 3375-3383.

Sawiak, S.J., Wood, N.I., Carpenter, T.A., Morton, A.J., 2012. Huntington's disease mouse models online: high-resolution MRI images with stereotaxic templates for computational neuroanatomy. PLoS One 7, e53361.

Sawiak, S.J., Wood, N.I., Williams, G.B., Morton, A.J., Carpenter, T.A., 2009a. Use of magnetic resonance imaging for anatomical phenotyping of the R6/2 mouse model of Huntington's disease. Neurobiol Dis 33, 12-19.

Sawiak, S.J., Wood, N.I., Williams, G.B., Morton, A.J., Carpenter, T.A., 2009b. Voxel-based morphometry in the R6/2 transgenic mouse reveals differences between genotypes not seen with manual 2D morphometry. Neurobiol Dis 33, 20-27.

Sawiak, S.J., Wood, N.I., Williams, G.B., Morton, A.J., Carpenter, T.A., 2013. Voxel-based morphometry with templates and validation in a mouse model of Huntington's disease. Magn Reson Imaging 31, 1522-1531.

Slow, E.J., van Raamsdonk, J., Rogers, D., Coleman, S.H., Graham, R.K., Deng, Y., Oh, R., Bissada, N., Hossain, S.M., Yang, Y.Z., Li, X.J., Simpson, E.M., Gutekunst, C.A., Leavitt, B.R., Hayden, M.R., 2003. Selective striatal neuronal loss in a YAC128 mouse model of Huntington disease. Hum Mol Genet 12, 1555-1567.

Walker, F.O., 2007. Huntington's disease. Lancet 369, 218-228. 


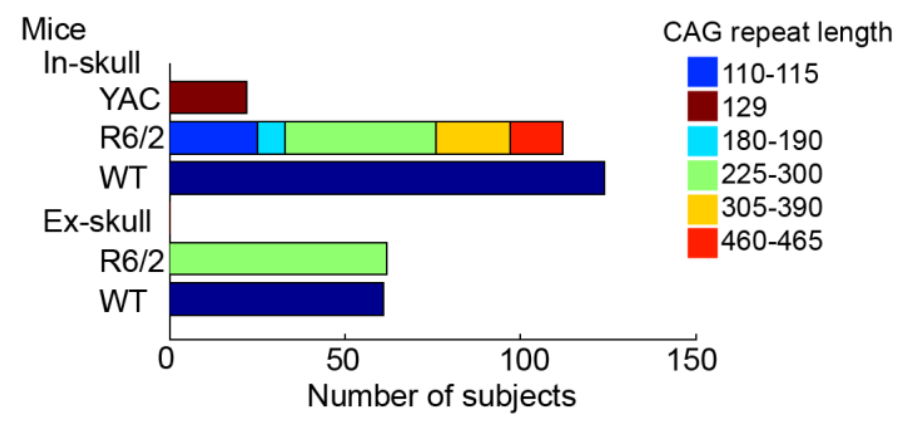

Figure 1 Breakdown of mice available in our repository to date. CAG repeat length is indicated for R6/2 mice (super short 109-114, short 182-187, medium 224-299, long 305-391 and super long 459464).

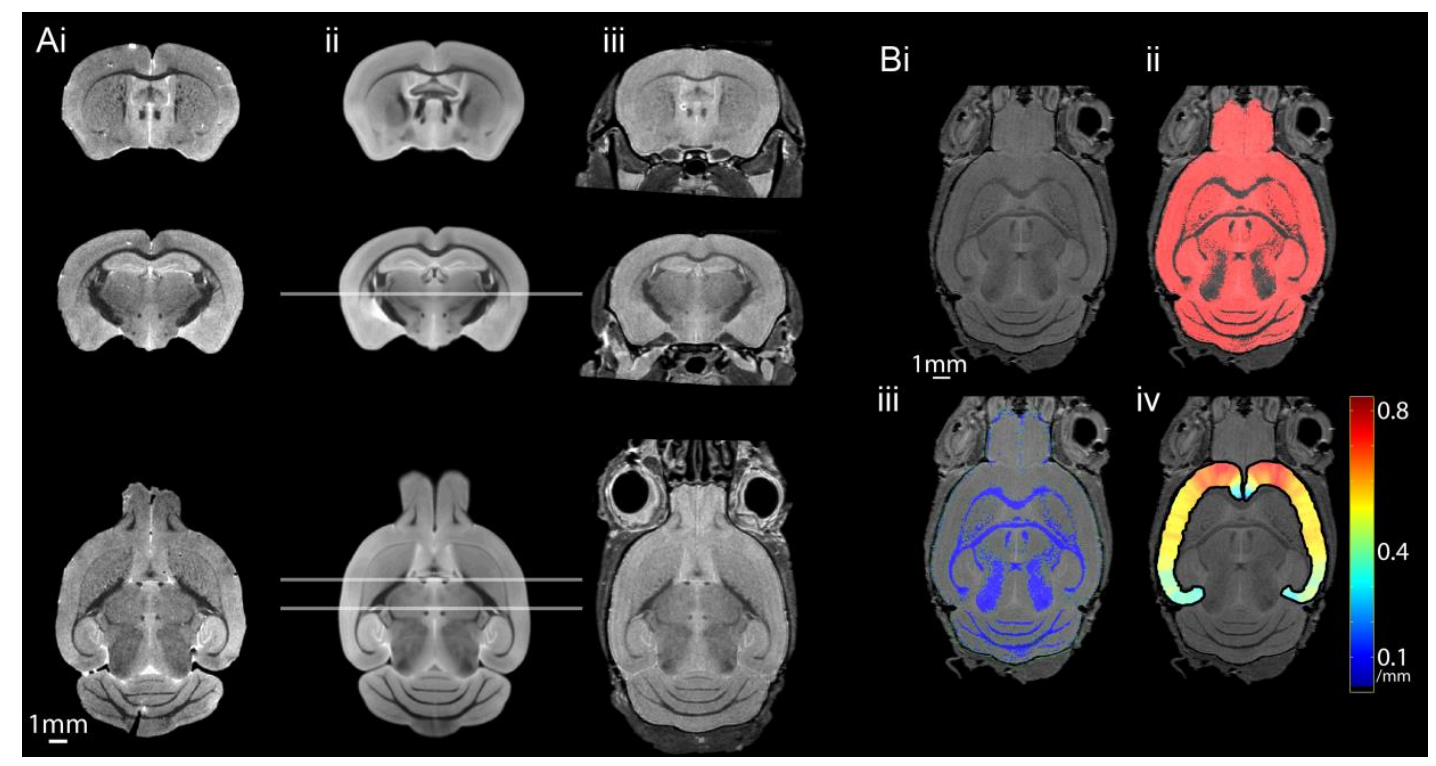

Figure 2 Exemplar data from the library. Ex-skull (Ai) and in-skull (Aiii) data are shown with the mouse brain atlas (Aii). A typical set of processed data including grey-matter maps (Bii), whitematter maps (Biii) and cortical thickness maps (Biv) are also shown. 


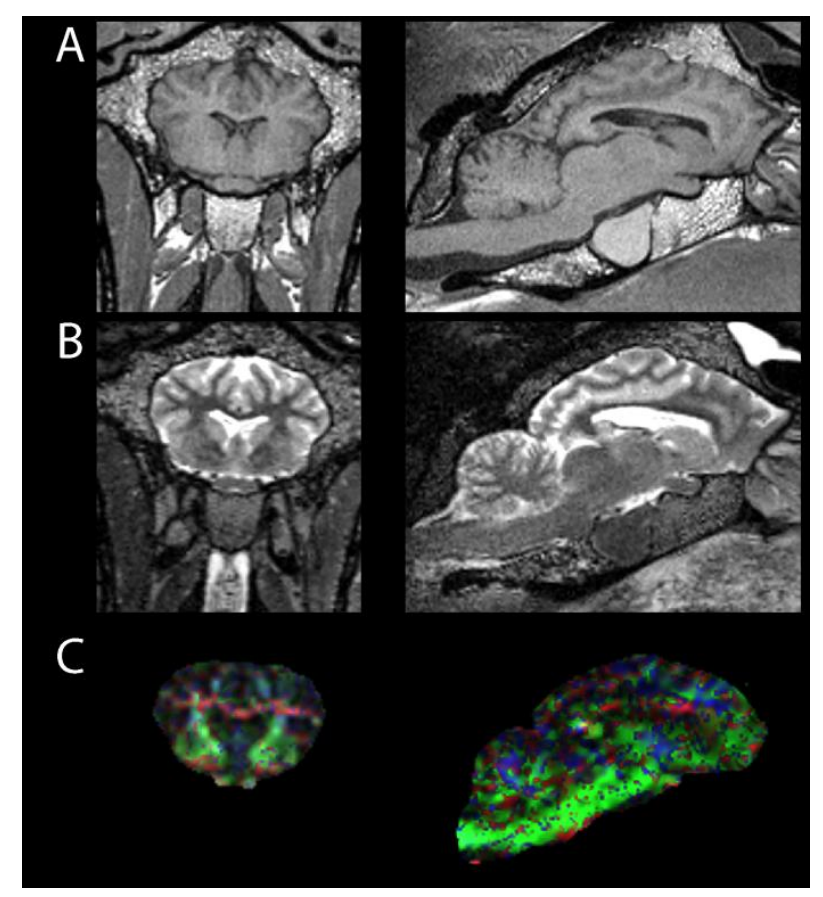

Figure 3: Coronal and sagittal sections from a typical sheep dataset in the library with (A) T1weighted contrast, (B) T2-weighted contrast and (C) coloured fractional anisotropy map calculated from DTI data. 